\title{
BM] Global Health Are there sex differences in completeness of death registration and quality of cause of death statistics? Results from a global analysis
}

\author{
Tim Adair (D , ${ }^{1}$ U S H Gamage, ${ }^{1}$ Lene Mikkelsen, ${ }^{1}$ Rohina Joshi ${ }^{2,3}$
}

To cite: Adair T, Gamage USH, Mikkelsen L, et al. Are there sex differences in completeness of death registration and quality of cause of death statistics? Results from a global analysis. BMJ Global Health 2021;6:e006660. doi:10.1136/ bmjgh-2021-006660

Handling editor Seye Abimbola

- Additional supplemental material is published online only. To view, please visit the journal online (http://dx.doi.org/10. 1136/bmjgh-2021-006660).

Received 18 June 2021 Accepted 11 September 2021

Check for updates

(C) Author(s) (or their employer(s)) 2021. Re-use permitted under CC BY-NC. No commercial re-use. See rights and permissions. Published by BMJ.

${ }^{1}$ Melbourne School of Population and Global Health, The

University of Melbourne, Carlton, Victoria, Australia

${ }^{2}$ The George Institute for Global Health, Newtown, New South

Wales, Australia

${ }^{3}$ The George Institute for Global Health, New Delhi, India

Correspondence to

Tim Adair;

timothy.adair@unimelb.edu.au

\section{ABSTRACT}

Introduction Recent studies suggest that more male than female deaths are registered and a higher proportion of female deaths are certified as 'garbage' causes (ie, vague or ill-defined causes of limited policy value). This can reduce the utility of sex-specific mortality statistics for governments to address health problems. To assess whether there are sex differences in completeness and quality of data from civil registration and vital statistics systems, we analysed available global death registration and cause of death data.

Methods Completeness of death registration for females and males was compared in 112 countries, and in subsets of countries with incomplete death registration. For 64 countries with medical certificate of cause of death data, the level, severity and type of garbage causes was compared between females and males, standardised for the older age distribution and different cause composition of female compared with male deaths.

Results For 42 countries with completeness of less than 95\% (both sexes), average female completeness was 1.2 percentage points (p.p.) lower (95\% uncertainty interval (UI) -2.5 to -0.2 p.p.) than for males. Aggregate female completeness for these countries was 7.1 p.p. lower ( $95 \% \mathrm{UI}-12.2$ to -2.0 p.p.; female $72.9 \%$, male $80.1 \%$ ), due to much higher male completeness in nine countries including India. Garbage causes were higher for females than males in 58 of 64 countries (statistically significant in 48 countries), but only by an average 1.4 p.p. (1.3-1.6 p.p.); results were consistent by severity and type of garbage.

Conclusion Although in most countries analysed there was no clear bias against females in death registration, there was clear evidence in a few countries of systematic undercounting of female deaths which substantially reduces the utility of mortality data. In countries with cause of death data, it was only of marginally poorer quality for females than males.

\section{INTRODUCTION}

Routine and high quality data on the fact and cause of death from a civil registration and vital statistics (CRVS) system should be the primary source of data to provide evidence

\section{Key questions}

What is already known?

- Recent studies have suggested that there is gender bias in civil registration and vital statistics (CRVS) systems, with less female than male deaths registered and female cause of death data of poore quality.

What are the new findings?

- In the 42 countries (of a total 112) where death registration was not complete, that is, less than $95 \%$, average completeness was 1.2 percentage points lower for females than males.

- In nine of these 42 countries, female completeness was at least 5 percentage points lower than for males, contributing to the aggregate female completeness for the 42 countries to be 7 percentage points lower than for males.

- For the quality of the cause of death data, garbage causes were consistently higher for females than males, but only by an average 1.4 percentage points.

What do the new findings imply?

- In most countries analysed, there is no evidence of a strong bias against females in death registration nor in the quality of the cause of death data. However, the data did also show that in a small number of countries such biases do exist, which severely reduces the utility of vital statistics in these countries.

for health policy and monitor progress to national and international health goals. ${ }^{1}$ In particular, accurate statistics on the completeness of registration and differences in this metric between males and females are important given that sex disparities exist in many key morbidity and mortality indicators. ${ }^{2}$ CRVS data also are important for legal and administrative purposes, providing families with a certificate of the evidence of death and, in many countries, the cause as ascertained by a physician using an International Medical Certificate of Cause of Death (MCCOD). 
Recent studies have suggested that the quality of CRVS data for female deaths are poorer than for male deaths, presenting evidence that fewer female than male deaths are registered. ${ }^{34}$ This, however, is clouded by the fact that globally about $20 \%$ more males than females die each year. ${ }^{2}$ There are some reasons though why there may be differences in the completeness of death registration by sex. Gender biases within a country (ie, the social construct of sex) that are represented by social and cultural factors can interact with characteristics of national legal and death registration systems to create barriers and power imbalances against females. ${ }^{3}$ For instance, most legal systems incentivise the registration of deaths by demanding a death certificate to allow the transfer of ownership of property and other assets, which often are predominantly owned by males. ${ }^{3}$ This can create a greater incentive for the registration of a male than female death, and likely contribute to higher levels of male than female completeness of registration (as a percentage of total deaths). Also, in some countries, more male than female deaths occur in hospitals, where deaths are more likely to be registered or reported; this may also lead to sex differences in death registration. ${ }^{5}$

The MCCOD is the primary source of information on causes of death globally. The attending physician completes a MCCOD with the sequence of events that led to death, often based on a review of the medical records, including imaging, pathology and laboratory investigations. ${ }^{6}$ In most countries, the certifying doctor has a professional and legal responsibility to ensure that the MCCOD is completed accurately. A significant challenge with cause of death data is that not all deaths are medically certified, and those which are can be biased due to poor certification practices that result in ill-defined or 'garbage' causes, which comprise a range of diagnoses that are of no use for policy. ${ }^{7}$ Some examples of garbage causes include modes of death such as 'heart failure' or symptoms such as 'fever' which could be due to either pneumonia or cancer or many other diseases.

There is evidence from a number of studies that a gender bias exists in detection and treatment procedures for cardiovascular diseases and more generally in medical textbooks. ${ }^{4-11}$ Gender bias could negatively impact on the quality of cause of death statistics for females if, for example, the quality of medical diagnosis or clinical care provided is poorer than for males. A past analysis of data from the WHO Mortality Database does seem to demonstrate that deaths in females are slightly less likely to have accurate information on cause of death than deaths in males, with the proportion of female deaths having unusable or 'garbage' causes being 2-3 percentage points (p.p.) higher than for male deaths. ${ }^{4}$ Depending on prevailing gender norms and other cultural factors, it is to be expected that the tendency to misdiagnose and miscertify causes between sexes may also vary among countries.

Any sex differences in the completeness of death registration or the quality of cause of death data prevent governments from recognising problems and planning effective, gender-sensitive policies. ${ }^{4}$ However, since the evidence base for any real sex differences in more than a handful of countries is tenuous, this study analyses the situation for all countries where such data are available. The study does not seek to assess gender bias in diagnosis, care or outcome, which is more suited to a smallscale rather than global analysis such as this. This study used completeness of registration because it is a superior measure of sex differences rather than the ratio of male to female deaths, which past analyses have relied on despite there being a greater number of male than female deaths in almost all countries, which will bias the results. ${ }^{24}$ For causes of death, we analysed both the extent of garbage causes, and also the severity and the type of garbage, and importantly we standardised for any bias in garbage causes due to the older age and different cause composition of female compared with male deaths.

\section{METHODS \\ Completeness of death registration}

We define completeness of death registration as the percentage of deaths in a population that are registered in the year they took place, divided by the total events that were estimated to have occurred.

For the assessment of completeness of death registration, we used publicly available national routine data of registered or reported deaths from various sources, including the Global Burden of Disease database and the WHO Mortality Database (see online supplemental table S1). ${ }^{12} 13$ Brazil and Peru are two countries where the data are from Ministry of Health databases and so are strictly 'reported' rather than 'registered' deaths; however, we use the term 'registered' deaths throughout the manuscript. The database for our study contains sexspecific death registration data of 112 countries from 2015 onwards, which excludes countries with less than 2000 annual estimated deaths because of uncertainty in estimates introduced by small numbers of deaths.

We measured completeness of death registration using the empirical completeness method, which uses inputs of registered crude death rate (registered deaths per 1000 population), under-five mortality rate, percentage of the population aged 65 years and above and completeness of under-five registration (age-specific registration data for children under 5 years where available), all data available or able to be calculated using registration data and data from the Global Burden of Disease (GBD) study. ${ }^{214}$ This method has been employed in numerous countries to estimate completeness separately for males and females. ${ }^{1516}$ The most recent year of available data for some countries does not disaggregate deaths by sex, so for these, we estimated the sex-specific completeness based on the last year for which data were available. In countries with high HIV mortality (Botswana, Lesotho and South Africa), we calculated completeness by using the GBD estimated total deaths as the denominator 


\section{Completeness}

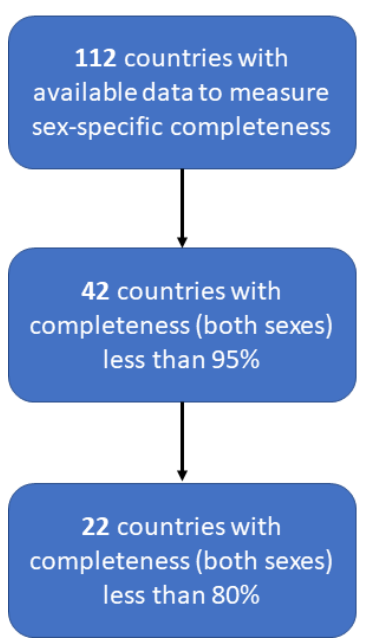

\section{Cause of death quality}

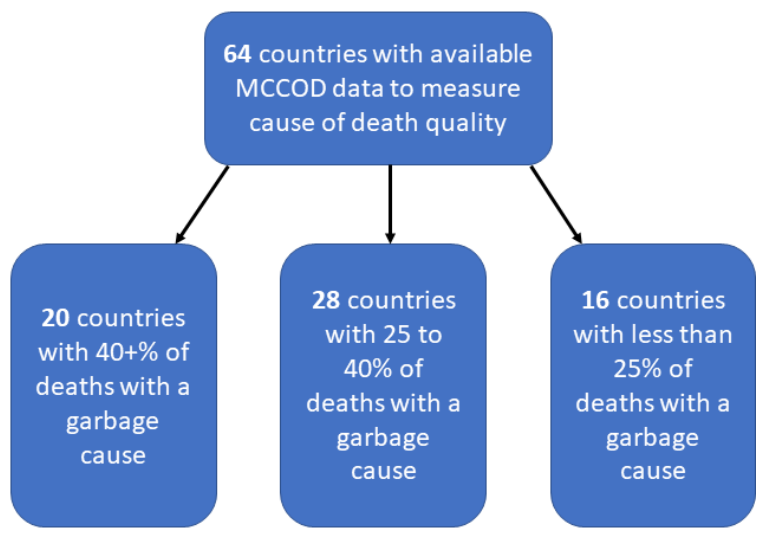

because the empirical completeness method is not suitable for measuring completeness in such countries. ${ }^{14}$

We focused our analysis on the 42 countries with less than 95\% completeness for both sexes and the 17 countries with less than $80 \%$ completeness for both sexes, because differences in completeness will less likely be present in countries with overall completeness approaching $100 \%$ (figure 1). We measured sex differences in completeness by aggregating data for countries in the data set; that is, weighting sex-specific completeness by the number of deaths in each country estimated by the GBD in $2019 .^{2}$ One issue with this calculation is that $79 \%$ of deaths in countries in our data set with less than 95\% completeness for both sexes occur in China and India. To enable comparison where those two countries do not dominate the results, we also calculated the average and median level of sex-specific completeness across countries; that is, by not weighting countries by the number of deaths (ie, treating each country equally irrespective of the number of deaths). Both absolute and relative differences in completeness between the sexes were calculated. We measured the number of additional female deaths that would be registered if females had the same levels of completeness as males, to understand which countries contributed most to sex differences in registration completeness. We also calculated the proportion of unregistered deaths that are male and female, to understand the extent to which future interventions to improve completeness should focus on specific sexes. The aggregate and country-level completeness figures, and differences between female and male completeness, are presented with $95 \%$ uncertainty intervals (UIs). The $95 \%$ UIs were calculated from 1000 simulations of predicted completeness incorporating uncertainty in the empirical completeness model and in the under-five mortality rate variable; for the three countries where the completeness was calculated using the GBD, we used their published 95\% UIs. We describe significant differences in completeness as being where the $95 \%$ UI of the difference in completeness does not overlap with zero.

\section{Garbage causes}

For measurement of sex differences in the percentage of deaths with a cause that is a garbage cause, we used data from the WHO Mortality Database, which contains data of the underlying cause of death coded to the 10th version of the International Classification of Diseases (ICD-10) by age group and sex. ${ }^{12}$ Similar to the previous analysis, we included deaths from 2015 onwards and countries with at least 2000 deaths per annum; this resulted in 64 countries available for analysis. Years of data used for each country for completeness and cause of death analyses is shown in online supplemental table S2.

The quality of the cause of death data was evaluated in terms of the extent of garbage causes in the data, using the same methodology and garbage code typologies as in the software ANACONDA (see online supplemental table S3 for further details). ${ }^{17-19}$ In the first typology investigating the type of error, if the proportions of total deaths in each error category are much higher for females than males, it might reflect gender biases and should be further investigated. Similarly, in the second typology where the garbage codes are grouped according to their severity (the potential impact they could have for misguiding policy), if the proportion of deaths in the 'very high' and 'high' categories are higher for females than males then this could be a sign of further gender bias beyond just the overall level of garbage. Other indicators of poor quality cause of death data are the percentage of deaths with an unspecified age or sex, and the percentage of deaths with a biologically implausible cause; we did not include these in our analysis because they comprised less than $1 \%$ of deaths in almost all countries in the database. ${ }^{20}$

In each country, we calculated the differences in the percentage of female deaths compared with male deaths that are in each garbage cause category after standardising for differences in age and cause distribution (six broad causes of death (group 1 causes (communicable, neonatal, maternal and nutritional) (ICD-10 codes 
A00-B99, O00-P99 and J00-J229), cardiovascular diseases (I00-I99), cancers (C00-D48), other non-communicable diseases (D49-H99, J23-N99 and Q00-Q99), injuries (S00T99, V00-Z99), R-coded (symptoms, signs and ill-defined conditions: R00-R99)) between the two populations. The standardisation of garbage was conducted separately for each country. The male garbage percentage was standardised to the female age-cause distribution of deaths in the country. That is, the male age-cause-specific garbage percentage was multiplied by the proportion of total female deaths that are in each age-cause grouping, and the sum of these age-cause-specific products is the age-cause standardised male garbage percentage. This standardisation is necessary to reduce potential bias in results due to the different age and cause structure of female and male deaths. It is well known that females are more likely to survive to older ages than males, where identifying causal pathways leading to death is a more challenging task for doctors than at younger ages. ${ }^{21}$ For example, garbage causes such as 'age-related physical debility' are more likely to be reported, while the heightened presence of comorbid conditions at these ages can lead to increased reporting of conditions that are not underlying causes of death, such as sepsis and heart failure. Standardising for cause is similarly important, as deaths from certain cause groups may be more likely in one sex and also more likely to result in a garbage cause (eg, 'heart failure' is commonly misclassified from cardiovascular diseases such as ischaemic heart disease). The analyses were conducted within different levels of overall garbage $(40+\%, 25<40 \%,<25 \%$; figure 1$)$. The $95 \%$ UIs of garbage were calculated from 1000 simulations that assume the garbage cause proportions have a binomial distribution. We also describe significant differences in garbage as being where its $95 \%$ UI does not overlap with zero.

\section{RESULTS}

\section{Completeness of registration}

For all 112 countries with data to enable calculation of sex-specific completeness, aggregate female completeness $(84.5 \%, 95 \%$ UI $82.2 \%-86.6 \%)$ is 3.2 p.p. lower (95\% UI -6.1 to -0.2 p.p.) than male completeness

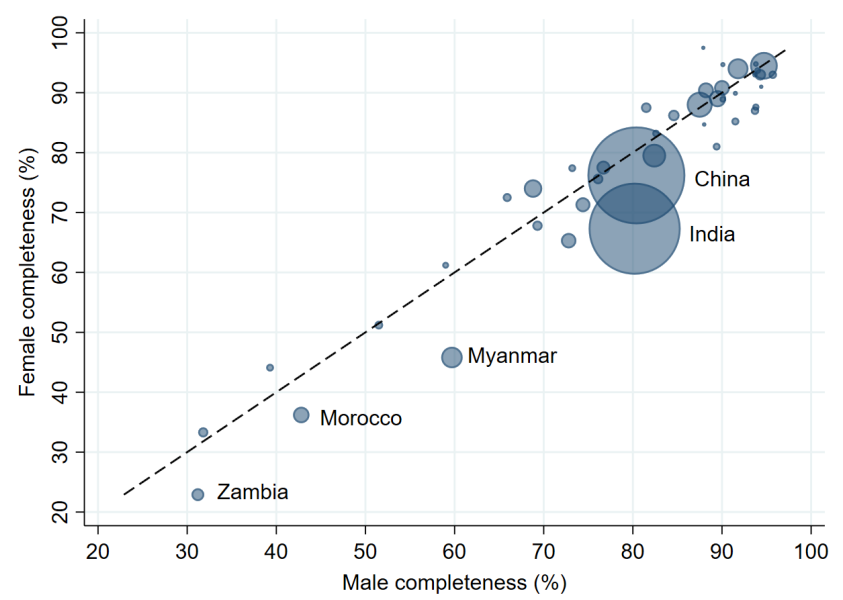

Figure 2 Female vs male completeness by country, countries with completeness for both sexes of less than $95 \%$. Dashed line indicates equality of male and female completeness. Marker size indicates estimated deaths in country in 2019.

$(87.7 \%, 85.5 \%-89.4 \%)$ (table 1, figure 2). When considering only the 42 countries with completeness for both sexes of less $95 \%$, the difference widens to 7.1 p.p. $(-12.2$ to -2.0 p.p.; female $72.9 \%$ ( $68.7 \%-79.6 \%)$, male $80.1 \%$ $(76.5 \%-83.0 \%))$. These results, however, are significantly impacted by India and China (the two largest markers in figure 2), which comprised almost $80 \%$ of deaths in 2019 in these 42 countries. For the 17 countries with completeness of less than $80 \%$, the female completeness is 8.1 p.p. lower ( -14.0 to -2.2 p.p.). Results for all countries are shown in online supplemental table S4A,B).

For the 42 countries with completeness of less than $95 \%$ for both sexes, females have only slightly lower registration completeness, being an average 1.2 p.p. lower $(-2.5$ to -0.2 p.p.) and a median 0.5 p.p. lower $(-1.9$ to -0.8 p.p.) (table 2). Of these 42 countries, female completeness is lower than male completeness in 25 countries, at least 5 p.p. lower in nine countries, but only significantly lower (ie, $95 \%$ UI of difference in completeness does not overlap with zero) in seven countries; female completeness is significantly higher in four countries (online supplemental table S4A). Including only countries with

Table 1 Summary results of male and female completeness (\%), aggregate analysis (ie, weighted by number of deaths in each country)

\begin{tabular}{llllll}
\hline & \multicolumn{2}{l}{ Completeness by sex (\%) } & & \\
\cline { 2 - 2 } Completeness (both sexes) & Female & & Male & Absolute difference† (p.p.) \\
\hline All countries* $(n=112)$ & $84.5(82.2-86.6)$ & $87.7(85.5-89.4)$ & & $-3.2(-6.1$ to -0.2$)$ \\
\hline Less than $95 \%(n=42)$ & $72.9(68.7-76.6)$ & $80.1(76.5-83.0)$ & & $-7.1(-12.2$ to -2.0$)$ \\
\hline Less than $80 \%(n=17)$ & $70.4(65.6-74.6)$ & $78.6(74.5-81.8)$ & & $-8.1(-14.0$ to -2.2$)$ \\
\hline
\end{tabular}

Results in parentheses are $95 \%$ uncertainty intervals. Over $90 \%$ of deaths in countries with completeness of less than $80 \%$ occur in China and India.

${ }^{*}$ Countries with sex-specific data.

†Female-male.

n, number of countries; p.p., percentage points. 
Table 2 Summary results of male and female completeness, country-level analysis (ie, not weighted by number of deaths in each country)

\begin{tabular}{|c|c|c|c|c|}
\hline \multirow[b]{2}{*}{ Completeness (both sexes) } & \multicolumn{2}{|c|}{ Average country completeness (\%) } & \multicolumn{2}{|c|}{ Absolute difference* (p.p.) } \\
\hline & Female & Male & Average & Median \\
\hline Less than $95 \%$ & $77.6(76.7-78.7)$ & $78.8(78.0-79.9)$ & $-1.2(-2.5$ to -0.2$)$ & $-0.5(-1.9$ to -0.8$)$ \\
\hline Less than $80 \%$ & $60.0(57.8-61.9)$ & $62.0(59.9-63.8)$ & $-2.0(-4.8$ to -0.9$)$ & $-0.4(-4.4$ to 3.6$)$ \\
\hline
\end{tabular}

Country-level average and median. Results in parentheses are 95\% uncertainty intervals.

${ }^{*}$ Female-male.

p.p., percentage points.

completeness of less than $80 \%$, the average absolute difference in completeness is 2.0 p.p. ( -4.8 to -0.9 p.p. $)$, with a median of 0.4 p.p. ( -4.4 to -3.6 p.p.); much lower than the completeness weighted by the number of deaths in each country.

The countries where female completeness is lowest compared with male completeness are Myanmar (-13.9 p.p., -25.2 to -1.6$)$, followed by India (-12.9 p.p., -22.8 to -3.5$)$, Libya -8.4 p.p. (-29.2 to 16.4$)$, Zambia $(-8.3$ p.p., -16.0 to -0.5$)$ and Nepal (-7.6 p.p., -14.2 to -0.9$)$ (table 3).

In countries with registration completeness of less than $95 \%$, if female deaths had the same level of completeness as males, there would be 790541 (223 886-1 370 427) more female deaths registered, or $9.6 \%$ more $(2.7 \%-$ $16.7 \%$; compared with 8.22 million) (online supplemental table S5). This difference is mostly attributable to India, where if female deaths had the same completeness as males, there would be 566780 more female deaths registered (153 136-1 011 116, or $72 \%$ of the total difference) (online supplemental table S5). Other countries where there would be a large increase in the absolute number of female registered deaths if they had the same completeness as males are in China (183 874 more, -185 360-547 899) and Myanmar (26 747, 3126-48 871). In several countries, there are more male than female deaths that are unregistered despite male completeness being similar or even higher than for females; this is because estimated total male deaths are higher than total female deaths. However, in India, $59 \%$ of unregistered deaths are female.

\section{Quality of cause of death data}

Female garbage is an average 1.4 p.p. higher (1.3-1.6 p.p.) than for males in the 64 countries with data, with a median of 1.4 p.p. (1.3-1.5 p.p.) (table 4, figure 3). The female garbage cause percentage is however higher in 58 countries and significantly higher in 48 countries (ie, $95 \%$ UI in the difference in garbage causes does not overlap with zero), with male garbage only higher in six countries and significantly higher in two countries. The highest sex difference is found in Malta where the female garbage is 5.5 p.p. (2.6-8.3 p.p.) higher than for males. The absolute difference between female and male garbage is similar irrespective of whether the level of garbage for both sexes is high or low. Full garbage results for all countries are presented in online supplemental tables S6, S7 and S8. The completeness of the MCCODs with the cause of death data, for each sex as a percentage of estimated deaths in the country, is above $90 \%$ for most countries and with minimal differences between males and females (online supplemental table S9).

The slightly higher female garbage is evenly distributed across the different levels of garbage severity, being relatively low in the 'very high' and low' categories compared with the contribution to differences in overall garbage

Table 3 Individual country completeness by sex, countries with female completeness at least 5 percentage points lower than for males

\begin{tabular}{lllll}
\hline Country & Year & Female (\%) & Male (\%) & Absolute difference* (p.p.) \\
\hline Myanmar & 2017 & $45.8(36.6-53.3)$ & $59.7(50.8-67.6)$ & $-13.9(-25.2$ to -1.6$)$ \\
\hline India & 2018 & $67.3(58.5-74.9)$ & $80.2(74.2-85.0)$ & $-12.9(-22.8$ to -3.5$)$ \\
Libya & 2017 & $81.0(63.8-92.6)$ & $89.4(73.6-98.1)$ & $-8.4(-29.2$ to 16.4$)$ \\
\hline Zambia & 2018 & $22.9(18.1-28.3)$ & $31.2(25.3-36.7)$ & $-8.3(-16.0$ to -0.5$)$ \\
Nepal & 2017 & $65.3(61.1-70.0)$ & $72.8(67.9-77.5)$ & $-7.6(-14.2$ to -0.9$)$ \\
Republic of Moldova & 2019 & $87.0(82.5-90.5)$ & $93.7(91.4-95.3)$ & $-6.7(-11.6$ to -2.6$)$ \\
Morocco & 2016 & $36.2(27.8-45.6)$ & $42.8(32.1-53.9)$ & $-6.6(-20.8$ to 7.8$)$ \\
\hline Mongolia & 2019 & $87.6(83.8-90.6)$ & $93.8(91.5-95.5)$ & $-6.3(10.4$ to -2.4$)$ \\
\hline Paraguay & 2017 & $85.2(79.4-89.2)$ & $91.5(87.6-94.1)$ & $-6.3(-12.4$ to -0.1$)$ \\
\hline
\end{tabular}

Results in parentheses are 95\% uncertainty intervals.

${ }^{*}$ Female-male.

p.p., percentage points. 
Table 4 Summary results of male and female garbage (age-cause standardised), country-level analysis

\begin{tabular}{|c|c|c|c|c|}
\hline \multirow[b]{2}{*}{ Garbage (both sexes) } & \multicolumn{2}{|c|}{ Average country garbage (\%) } & \multicolumn{2}{|c|}{ Absolute difference (p.p.)* } \\
\hline & Female & Male & Average & Median \\
\hline All $(n=64)$ & 34.7 (34.6-34.8) & $33.3(33.2-33.3)$ & $1.4(1.3-1.6)$ & $1.4(1.3-1.5)$ \\
\hline $40 \%+(n=20)$ & $51.3(51.1-51.4)$ & $49.7(49.6-49.9)$ & $1.5(1.3-1.7)$ & $1.4(1.2-1.7)$ \\
\hline $25<40 \%(n=28)$ & $32.8(32.6-33.0)$ & $31.1(30.9-31.3)$ & $1.7(1.4-1.9)$ & $1.4(1.2-1.6)$ \\
\hline$<25 \%(n=16)$ & $19.8(19.6-20.0)$ & $18.7(18.6-19.9)$ & $1.1(0.8-1.3)$ & $1.2(0.9-1.4)$ \\
\hline
\end{tabular}

Male garbage \% standardised to female age-cause death distribution. Country-level average and median. Results in parentheses are $95 \%$ uncertainty intervals.

${ }^{*}$ Female-male.

n, number of countries; p.p., percentage points.

(table 5). According to type of garbage, the majority of the higher female garbage is found in the categories 'Insufficiently specified' and 'Intermediate causes' (table 6), the two categories which also comprise most of the garbage.

\section{DISCUSSION}

This analysis provides evidence that in 42 countries with completeness of death registration for both sexes of less than $95 \%$, female completeness is only an average 1.2 p.p. lower than for males. In only nine out of the 42 countries completeness of death registration for females is at least 5 p.p. lower than for males, and in only seven countries where completeness is significantly lower. In these countries, completeness for males $(80 \%)$ was 7 p.p. higher than for females $(73 \%)$, mainly due to the significant differences found in some large countries, particularly India which accounts for almost three-quarters of the aggregate disparity. When restricting the analysis to countries with completeness of less than $80 \%$, aggregate completeness for females is 8 p.p. lower than for males, and an average 2.0 p.p. lower. Aggregate differences in completeness between females and males are only 3

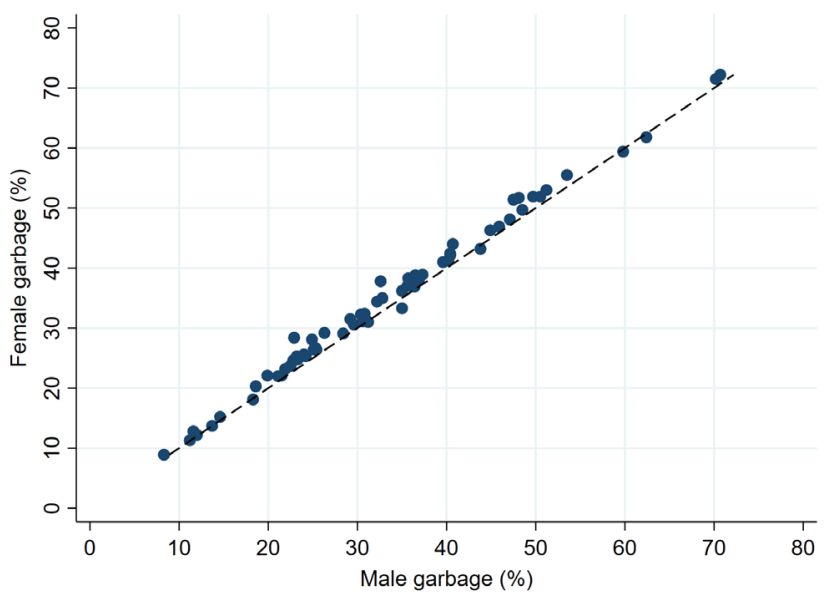

Figure 3 Female vs male garbage (age-cause standardised) by country. Dashed line indicates equality of male and female garbage. p.p. if all 112 countries with sex-disaggregated data are included.

Lower completeness of female compared with male death registration can reduce the value of sexdisaggregated mortality statistics that are evidence for governments of pressing health problems, for example excess mortality from COVID-19. To better understand how and why sex differences in death registration occur, future research should focus on investigating the sizeable sex differences that exist in a handful of countries. The sex differentials in death registration in specific countries are likely to have several origins that vary by country and which need to be investigated and documented to motivate government action to reduce them. In general, apart from inheritance, insurance and pension claims, there are few incentives to register a death, and in many countries, these incentives would more likely apply to male than female deaths. In addition, because of longer life expectancy, females often survive their husbands and become single households, which may mean there is no one to register their death. ${ }^{22}$ Another reason may be that in some countries a higher proportion of male deaths occurs in hospitals and therefore are certified and more likely to be registered than community deaths. ${ }^{5}$ Finally, a higher proportion of males than females die from injuries and assaults, a cause group commonly subject to police investigation and hence deaths that are more likely to be registered. ${ }^{23}$ The countries with much higher male than female completeness occur in a range of regions outside of Western Europe and North America, being found in populations in South Asia (India and Nepal), Southeast Asia (Myanmar), Central Asia (Mongolia), North Africa (Libya and Morocco), sub-Saharan Africa (Zambia), South America (Paraguay) and Eastern Europe (Republic of Moldova). Interestingly, not all these countries rank poorly on gender issues, and three (Moldova, Libya and Mongolia) are all ranked in the top 50\% of countries in the United Nations Development Programme's Gender Inequality Index. ${ }^{24}$

Analysis of cause of death data by sex for the 64 countries, standardised by age and cause, demonstrates that, on average, garbage causes of death are 1.4 p.p. higher in females compared with males. This slightly higher level of garbage 
Table 5 Female and male (age-cause standardised) garbage by severity, 64 countries

\begin{tabular}{|c|c|c|c|c|c|}
\hline All & All & Very high & High & Medium & Low \\
\hline Female average (\%) & 34.7 & 15.7 & 4.3 & 3.4 & 11.4 \\
\hline Male average (\%) & 33.3 & 15.3 & 3.9 & 3.1 & 11.0 \\
\hline Average difference* (p.p.) & 1.4 & 0.4 & 0.4 & 0.3 & 0.4 \\
\hline Median difference* (p.p.) & 1.4 & 0.2 & 0.4 & 0.3 & 0.3 \\
\hline
\end{tabular}

Male garbage \% standardised to female age-cause death distribution. Country-level average and median.

${ }^{*}$ Female-male.

p.p., percentage points.

causes among females is remarkably consistently across countries, with over $90 \%$ of countries having a higher fraction of female than male garbage in the cause of death data. There is no clear pattern in the sex differences by whether countries have high or low levels of garbage. Notably, the higher garbage of females is not systematically attributable to more severe levels of garbage nor the type of garbage. Previous research has identified a gender bias in both diagnosis and treatment procedures for cardiovascular diseases and nonreproductive organ cancers, but our data set was not able to identify the precise pathways that such biases would impact on sex differences in causes of death and so further research is needed in this area. ${ }^{925-27}$

The strengths of this analysis include using the largest currently available data set to evaluate global sex differences in completeness of death registration and pattern and extent of garbage coding, as well as standardising differences between sexes for any biases due to age and cause. While previous analyses have investigated overall differences in causes of death among males and females, our study has analysed the contribution of specific types of garbage to observed differences between males and females, thus providing more insight into sex differentials in mortality data. ${ }^{34}$

Our study has several limitations. First, many low-income and middle-income countries could not be included due to lack of access to their data or because the data are either not publicly available or do not exist. We only have sex-disaggregated death registration data for 112 countries and medically certified cause of death data for 64 countries, reflecting the poor state of death statistics globally and emphasising the need for improved availability of sex-disaggregated data. The countries for which we do not have data are disproportionately those that rank poorly on gender indicators, so whatever data they have available may show a worse picture on sex differences in mortality data than shown by our study. ${ }^{24}$ In India, MCCODs are only available for around one-fifth of all deaths (essentially hospital deaths), and $62 \%$ of MCCODs are for deaths of males, similar to what is found for registered deaths. ${ }^{528}$ Of the reported causes of death, the proportion assigned a cause in the R chapter of ICD (Symptom Signs and Abnormal Clinical Findings not elsewhere classified, ie, one component of garbage) is almost the same for males $(13 \%)$ and females (14\%). Due to only a small proportion of deaths being medically certified in such countries with large differences in registration completeness, the results may not reveal the full extent of sex disparities.

In recent years, several regional and global initiatives, including the Data for Health Initiative, have focused on improving death registration and cause of death certification. ${ }^{1}$ Initiatives such as these are important to ensure that countries have CRVS systems that are able to produce vital statistics of sufficient quality for policy use and that they do not discriminate against females. Reduction in true sex differences in death registration and the quality of cause of death data should be addressed by interventions, including removing disincentives such as a fee to register deaths and enabling registration closer to where people live. Countries that have an active process of initial notification of deaths by institutions or a formal agent, such as community health workers (which are mostly females), rather than relying on the family, are likely to increase death registration and reduce bias towards not registering female deaths. ${ }^{329}$ Advocacy and education about the importance of death registration will

Table 6 Female and male (age-cause standardised) garbage by type, 64 countries

\begin{tabular}{lllllcl}
\hline Country & All & $\begin{array}{l}\text { Symptoms, sign, } \\
\text { ill-defined }\end{array}$ & Impossible & Intermediate & $\begin{array}{c}\text { Insufficiently } \\
\text { Immediate }\end{array}$ & $\begin{array}{l}\text { specified } \\
\text { sped }\end{array}$ \\
\hline Female average (\%) & 34.7 & 4.8 & 1.6 & 11.8 & 1.5 & 15.0 \\
Male average (\%) & 33.3 & 4.9 & 1.7 & 11.2 & 1.5 & 14.0 \\
Average difference* (p.p.) & 1.4 & 0.0 & 0.0 & 0.6 & -0.1 & 1.0 \\
Median difference* (p.p.) & 1.4 & 0.0 & 0.0 & 0.4 & 0.0 & 0.9 \\
\hline
\end{tabular}

Male garbage \% standardised to female age-cause death distribution. Country-level average and median. Further information about types of garbage are shown in online supplemental table S3.

${ }^{*}$ Female-male.

p.p., percentage points. 
raise awareness and interest of its benefits other than just for transfer of assets (which disproportionately favours males in some countries). Also, training doctors in medical certification of causes of death will decrease the proportion of garbage causes, especially originating from heart diseases, and thereby disproportionately improve the quality of information on the causes of female deaths. In countries where a high proportion of deaths occur at home, low-cost interventions such as linking notification of the fact of death to registration and initiation of a verbal autopsy interview will help promote death registration, including that of females. ${ }^{30} 31$ Increases in registration of male deaths are also important to improve overall levels of completeness, especially given that our analysis showed that in many countries more male than female deaths are unregistered. To improve the use of death registration data, countries should also publish all mortality indicators disaggregated by sex, where possible.

\section{CONCLUSION}

Our study did not find evidence of a systematic and significant bias in registering female compared with male deaths, except in seven countries. In these countries, the utility of the vital statistics for policy and planning is severely reduced because of undercounting of female deaths. We also did not find, at the global level, any clear difference in the quality of cause of death data between males and females, irrespective of the level of the proportion of garbage causes. Given the current global drive to improve CRVS systems, availability of sex-disaggregated death registration and MCCOD data in more countries will enable an analysis such as this to better reflect and monitor potential gender bias in global deaths. Future analyses should also seek to minimise the influence of other factors, such as the differing age and cause structure of male and female deaths, to assess the true situation of sex differences in death registration and cause of death data quality.

Contributors TA developed the analytical methods, conducted the data analyses, contributed to the drafts and edited the final draft. USH contributed to the drafts and edited the final draft. LM contributed to interpretation of the data and made substantive edits to the drafts. RJ conceptualised the study, contributed to the drafts and edited the final draft.

Funding This study was funded under an award from Bloomberg Philanthropies and the Australian Department of Foreign Affairs and Trade to the University of Melbourne to support the Data for Health Initiative. The funders had no role in study design, data collection and analysis, decision to publish or preparation of the manuscript. RJ is supported by a National Heart Foundation Fellowship and a Scientia Fellowship from UNSW.

Competing interests None declared.

Patient consent for publication Not required.

Provenance and peer review Not commissioned; externally peer reviewed.

Data availability statement All data relevant to the study are included in the article or uploaded as supplementary information.

Supplemental material This content has been supplied by the author(s). It has not been vetted by BMJ Publishing Group Limited (BMJ) and may not have been peer-reviewed. Any opinions or recommendations discussed are solely those of the author(s) and are not endorsed by BMJ. BMJ disclaims all liability and responsibility arising from any reliance placed on the content. Where the content includes any translated material, BMJ does not warrant the accuracy and reliability of the translations (including but not limited to local regulations, clinical guidelines, terminology, drug names and drug dosages), and is not responsible for any error and/or omissions arising from translation and adaptation or otherwise.

Open access This is an open access article distributed in accordance with the Creative Commons Attribution Non Commercial (CC BY-NC 4.0) license, which permits others to distribute, remix, adapt, build upon this work non-commercially, and license their derivative works on different terms, provided the original work is properly cited, appropriate credit is given, any changes made indicated, and the use is non-commercial. See: http://creativecommons.org/licenses/by-nc/4.0/.

\section{ORCID iD}

Tim Adair http://orcid.org/0000-0002-1562-4452

\section{REFERENCES}

1 Lopez AD, McLaughlin D, Richards N. Reducing ignorance about who dies of what: research and innovation to strengthen CRVS systems. BMC Med 2020;18:58.

2 GBD 2019 Demographics Collaborators. Global age-sexspecific fertility, mortality, healthy life expectancy (HALE), and population estimates in 204 countries and territories, 1950-2019: a comprehensive demographic analysis for the global burden of disease study 2019. Lancet 2020;396:1160-203.

3 AbouZahr C, Joshi R, Thomas J. Making civil registration and vital statistics systems work for women. knowledge brief series on gender and CRVS: centre of excellence for civil registration and vital statistics (CRVS) systems, 2019.

4 Cobos Muñoz D, Sant C, Revenga Becedas R. The dangers of gender bias in CRVS and cause of death data: the path to health inequality. Centre of Excellence for Civil Registration and Vital Statistics (CRVS) Systems, International Development Research Centre, Ottawa, Ontario: Knowledge Brief Series on Gender and CRVS, 2020.

5 Office of the Registrar General India. Report on medical certification of cause of death, 2018. New Delhi: Office of the Registrar General India, Ministry of Home Affairs, Vital Statistics Division, 2020.

6 Rampatige R, Gamage S, Peiris S, et al. Assessing the reliability of causes of death reported by the vital registration system in Sri Lanka: medical records review in Colombo. Health Inf Manag 2013;42:20-8.

7 lburg KM, Mikkelsen L, Adair T, et al. Are cause of death data fit for purpose? Evidence from 20 countries at different levels of socioeconomic development. PLoS One 2020;15:e0237539.

8 Aggarwal NR, Patel HN, Mehta LS, et al. Sex differences in ischemic heart disease: advances, obstacles, and next steps. Circ Cardiovasc Qual Outcomes 2018;11:e004437.

9 Daugherty SL, Blair IV, Havranek EP, et al. Implicit gender bias and the use of cardiovascular tests among cardiologists. J Am Heart Assoc 2017;6.

10 Dijkstra AF, Verdonk P, Lagro-Janssen ALM. Gender bias in medical textbooks: examples from coronary heart disease, depression, alcohol abuse and pharmacology. Med Educ 2008;42:1021-8.

11 Gudnadottir GS, Andersen K, Thrainsdottir IS, et al. Gender differences in coronary angiography, subsequent interventions, and outcomes among patients with acute coronary syndromes. Am Heart J 2017; 191:65-74.

12 World Health Organization. WHO mortality database. Geneva, 2021.

13 Global Burden of Disease Study. Global Durden of Disease deaths database. Seattle: Global Burden of Disease, 2020.

14 Adair T, Lopez AD. Estimating the completeness of death registration: an empirical method. PLoS One 2018;13:e0197047.

15 Shawon MTH, Ashrafi SAA, Azad AK, et al. Routine mortality surveillance to identify the cause of death pattern for out-of-hospital adult (aged 12+ years) deaths in Bangladesh: introduction of automated verbal autopsy. BMC Public Health 2021;21:491.

16 Zeng X, Adair T, Wang L, et al. Measuring the completeness of death registration in 2844 Chinese counties in 2018. BMC Med 2020;18:176.

17 Mikkelsen L, Moesgaard K, Hegnauer M, et al. ANACONDA: a new tool to improve mortality and cause of death data. BMC Med 2020;18:61.

18 Naghavi M, Richards N, Chowdhury H, et al. Improving the quality of cause of death data for public health policy: are all 'garbage' codes equally problematic? BMC Med 2020;18:55

19 Mikkelsen L, Richards N, Lopez A. Redefining 'garbage codes' for public health policy: Report on the expert group meeting, 27-28 February 2017. CRVS best-practice and advocacy. Melbourne: Australia University of Melbourne, 2019. 
20 Phillips DE, Lozano R, Naghavi M, et al. A composite metric for assessing data on mortality and causes of death: the vital statistics performance index. Popul Health Metr 2014;12:14.

21 Filippatos G, Andriopoulos P, Panoutsopoulos G, et al. The quality of death certification practice in Greece. Hippokratia 2016;20:19-25.

22 Yumiko K, Hertog S. Measuring household and living arrangements of older persons around the world: the United nations database on the households and living arrangements of older persons 2019. New York: United Nations, Department of Economics and Social Affairs, Population Division, Technical Paper, 2020.

23 Global Burden of Disease Collaborative Network. Global Burden of Disease study 2019 (GBD 2019) results. Seattle 2020.

24 United Nations Development Programme. The 2020 human development report. in: programme UND. New York: United Nations Development Programme, 2020.

25 Herold AH, Riker Al, Warner EA, et al. Evidence of gender bias in patients undergoing flexible sigmoidoscopy. Cancer Detect Prev 1997;21:141-7.
26 Sarasqueta C, Zunzunegui $M^{a}$ Victoria, Enríquez Navascues JM, et al. Gender differences in stage at diagnosis and preoperative radiotherapy in patients with rectal cancer. BMC Cancer 2020;20:759.

27 Beery TA. Gender bias in the diagnosis and treatment of coronary artery disease. Heart Lung 1995;24:427-35.

28 Office of the Registrar General India. Vital statistics of India based on the civil registration system, 2018. New Delhi: Office of the Registrar General India, Ministry of Home Affairs, Vital Statistics Division, 2020.

29 Cobos Muñoz D, Abouzahr C, de Savigny D. The 'Ten CRVS Milestones' framework for understanding Civil Registration and Vital Statistics systems. BMJ Glob Health 2018;3:e000673.

30 Adair T, Rajasekhar M, Bo KS, et al. Where there is no hospital: improving the notification of community deaths. BMC Med 2020;18:65

31 Hazard RH, Buddhika MPK, Hart JD, et al. Automated verbal autopsy: from research to routine use in civil registration and vital statistics systems. BMC Med 2020;18:60. 